\title{
Hillock and island formation during annealing of gold films
}

\author{
M S RAHMAN KHAN \\ Department of Applied Physics and Electronics, University of Rajshahi, Rajshahi, \\ Bangladesh \\ MS received 2 June 1986; revised 16 February 1987
}

\begin{abstract}
Kinetics of the hillock and island formation during annealing of thin gold film has been studied. The reorganisation of gold film has been distinguished in five stages in progression from the original film to the final island structure. The area occupied by the holes remained virtually constant until the removal of the metal from the substrate started. No increase in the number of holes was detected in the induction period. Hole densities decrease with increasing film thickness. Agglomerates were frequently found at the side of an enlarging hole. The hole growth rate increased steadily with temperature. The growth rate of holes eventually leads to a network structute. The catchment area increased with increasing initial film thickness.
\end{abstract}

Keywords. Hole density; Catchment area; delineation of Catchment area; hillock and island formation.

\section{Introduction}

The factors affecting the sintering of supported gold catalysts during catalytic oxidation are interesting and is worthy of study. But only a few observations exist of the mode of surface reorganisation in evaporated films, namely, hillock growth (Neilson et al 1967; Caswell et al 1963; Lahiri 1970; Gangulee 1970). Changes in morphology and the kinetics of agglomeration could be studied as the system is suitable for the study of the energetics and mechanism of mass transfer in supported metals. Microscopic examination of the agglomeration could clarify the relationship between the metal redistribution and the initial structure of evaporated films.

Surface diffusion-controlled space charges have been studied for thin films of gold (Brandon and Bradshow 1966). The decay of two types of step and growth of holes was observed. Caswell et al (1963) found that on annealing at $560^{\circ} \mathrm{C}$ in nitrogen for 20 min, hillocks were produced on the surface. Two different accounts have been given for hillock growth, both in terms of stress relief and the driving force. Existing work on the surface morphology annealing effects in thin films is not extensive and although some indication of the factors controlling the kinetics may be inferred, the lack of sufficient work describing the structure and energetics of such processes is apparent (Jorgensen and Wehner 1963). Two main classes of effect have been observed-agglomeration into discrete islands and surface hillock growth (Gimpi et al 1964). Island formation was found to occur whenever silver film on collodion support was heated to a sufficient temperature (Borel 1955). A detailed study of the metal reorganisation has been undertaken and the kinetics of the hillock and island formation has been reported in this paper.

\section{Experimental details}

Films were prepared by evaporation of gold wire $(99.99 \%$ purity) from a tungsten 
filament at a pressure of about $5 \times 10^{-0}$ torr. The evaporation rate was $9 \mathrm{~nm} / \mathrm{min}$. The films were deposited on freshly cleaved rocksalt, carbon-coated copper grids and silica, maintained either at room temperature or at an elevated temperature. The evaporation distance was kept at about $15 \mathrm{~cm}$ to ensure uniform film thickness. For transmission electron microscopy, specimens were coated with $10 \mathrm{~nm}$ carbon films by vacuum evaporation onto the gold deposit. The silica substrate was then dissolved in $30 \%$ hydrofluric acid and the carbon replicas were washed and mounted on copper grids.

Films on the copper grids were transferred through the atmosphere to the annealing system where they were placed in a small pyrex chamber through a polished conical glass seal. After closing the chamber, flushing of the annealing atmosphere for $15 \mathrm{~min}$ at a flow rate of $10-15 \mathrm{ml} / \mathrm{min}$ was sufficient to obtain a reproducible sintering behaviour. The pyrex chamber was then heated by insertion into a furnace. The sintering of films was studied by periodic cooling and removal of the samples. Cylindrical grade gases were used in all the cases. Below $10^{-2}$ torr of oxygen a vacuum system with air injector was used and the specimen was heated in a specially designed radiant heater within the system. The thickness of the deposited gold was measured by Tolansky's multiple beam interferometric method. The films were examined in a transmission electron microscope (Hitachi 500).

\section{Results}

It is convenient to distinguish five stages in the progression from the initial film to the final island structure. It should be noted that the changes in microstructure follow from the redistribution of a constant amount of material. Four features of the microstructure of the initial film were examined. The first of these, the hole density, was measured from transmission electron micrographs of the films in the thickness range $30-120 \mathrm{~nm}$ at a magnification of 15,000 . The hole density was calculated manually over a measured area. The smaller holes were occasionally indistinguishable from multigrain boundary intersections in the thinnest films. However, this was not a serious problem for the range of thickness considered above. Hole densities were found to decrease steadily with increasing film thickness (figure 1). The mean crystallite size was $4.5 \mathrm{~nm}$ for $100 \mathrm{~nm}$ film.

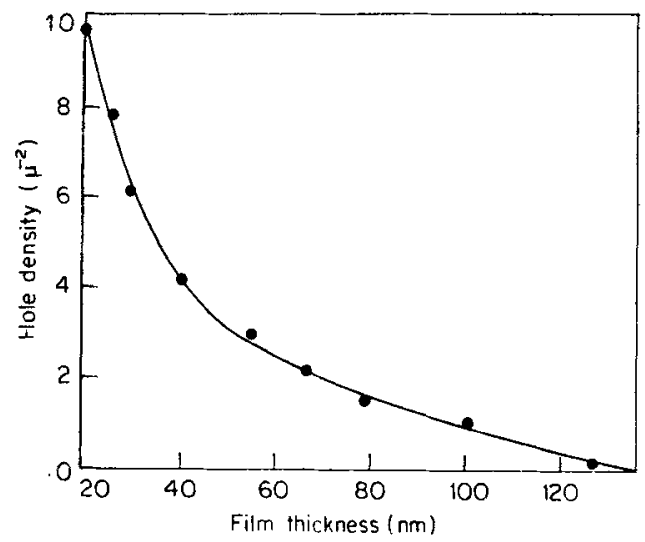

Figure 1. Dependence of hole density on film thickness. 


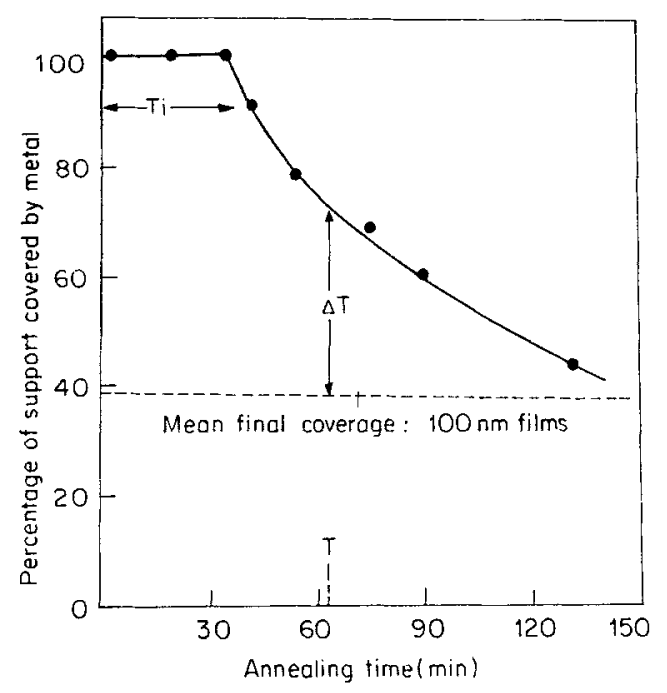

Figure 2. Variation in gold-supported area with annealing time.

Transmission electron micrographs of oxygen-annealed films showed little change initially from their original structure. The area occupied by holes remained virtually constant until removal of metal from the substrate started (figure 2). This interval is called the induction period. No increase in the number of holes was detected in this period. The length of the induction period Ti (min) was found to depend on the temperature $T(\mathrm{~K})$, the film thickness $d(\AA)$ and the gas environment. Agglomerates were frequently found at the side of an enlarging hole. The growing holes were irregular in shape, with multiple cups pointing upwards. The hole growth rate was measured at temperatures of $150-250^{\circ} \mathrm{C}$ and found to increase steadily with temperature. The growth of holes eventually leads to a network structure. Where cups at adjacent holes are close, a neck is effectively present and progressive thinning of these necks results in parting of the agglomerates into discrete islands. In some specimens, a population of much smaller islands was left between the main masses, probably by adhesion of metal to small areas of the intervening substrate. Prolonged annealing of the separated islands allowed them to slowly approach an equilibrium shape. Heating at $350^{\circ} \mathrm{C}$ in oxygen for about $48 \mathrm{hr}$ proved sufficient to allow islands of $<1 \mu$ diameter to attain an approximately hemispherical character. Transmission electron microscopy did not detect grain boundaries in most islands of this size; when viewed in true dark field, a few individual islands at a time were visible by transmission through a narrow band around their complete perimeter for each alignment of the illuminating beam.

Final island densities were counted visually from micrographs for a series of films of initial thickness of $30-120 \mathrm{~nm}$ heated in oxygen at $200^{\circ} \mathrm{C}$ for several hours. The island densities decreased steadily with increasing film thickness. Only the main population of large islands was introduced; islands left behind at a later stage by adhesion to points on the substrate were thus excluded; some estimation was involved at this point. A catchment area $A_{c}\left(\mu^{2}\right)$ was defined as the main area from which a given island had withdrawn material for growth; figure 3 shows a plot of $\log _{10} A_{c}\left(\mu^{2}\right)$ against $\log _{10} d(\mathrm{~nm})$. The catchment area increased steadily with increasing initial 


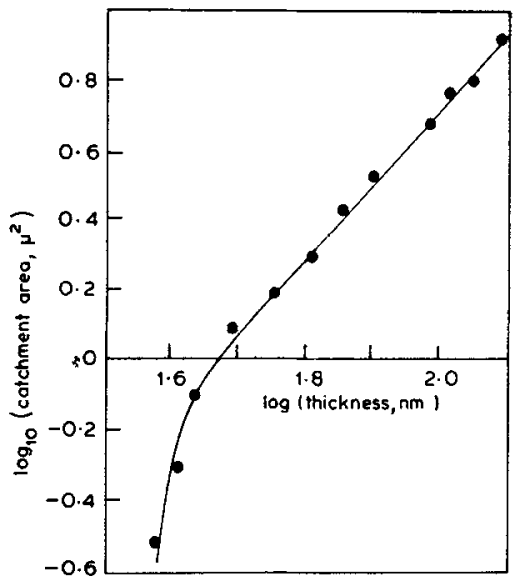

Figure 3. Relation between catchment area and initial film thickness.

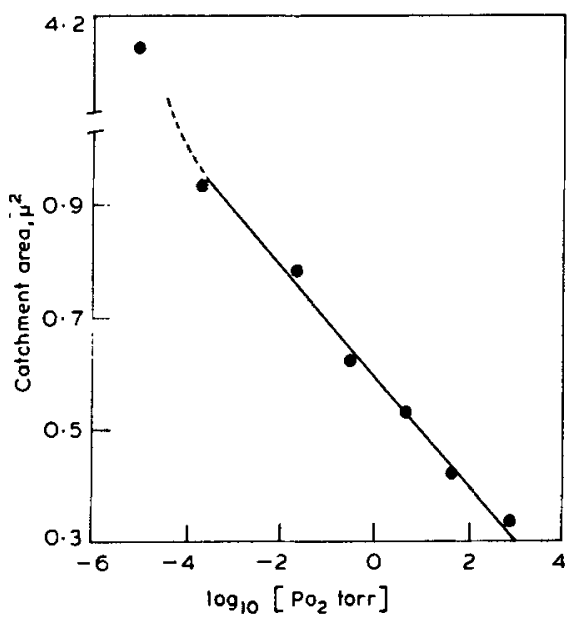

Figure 4. Relation between catchment area and oxygen partial pressure.

film thickness for all but the thinnest films and the points lie about a straight line of slope $2 \cdot 21$ indicating a linear relationship.

$$
\begin{aligned}
& \log _{10} A_{c}=2 \cdot 13 \log _{10} d-5 \cdot 31 \\
& \text { or } \quad A_{c}=4.53 \times 10^{-6} d^{2} .
\end{aligned}
$$

The effect of oxygen partial pressure on the final island density was also determined. The results shown in figure 4 reveal that the catchment area increase as $P_{\mathbf{O}_{2}}$ decreases. 


\section{Discussion}

The islands formed on annealing are so much larger than the crystallites in the initial film that the area from which they draw material cannot have been simply delimited by grain boundaries. The catchment area can be related to the properties of the original film. The areas form a network of adjacent polygons with varying numbers of side but with little error their dimensions may be represented conveniently as the radii, $r$ of the tangent circles. Hence,

$$
r=\left(A_{c} / \pi\right)^{\frac{1}{2}}
$$

and so from (1), $A_{c}=4.53 \times 10^{-6} d^{2}, r$ should be proportional to $d$.

An overall picture of catchment areas in relation to film structure can now be represented (figure 5). The formation of discrete solid nuclei from the vapour followed by growth until holes left between the enlarged islands (Pashley et al 1964) is presented diagrammatically in figure 5a. The delineation of a catchment area by such holes is shown in figure $5 \mathrm{~b}$. During annealing, these delineating holes effectively close up to cut off these areas of deposit which form the final islands. The lines joining the holes cover the deposit in an irregular network. In the case of both a regular and an irregular network, the number of areas thus delimited approaches the number of holes $n$ as $n \rightarrow \infty$. We must therefore assume that in the present case, one hole is associated with each catchment area. If $C=$ islands/initial hole, then it was observed experimentally that $I / C=6$ holes were associated with each island formed. Since only one hole per island can be on the lattices delineating area, we conclude that only about 1 in 6 of the observed holes is effective.

The temperature dependence of the length of the induction period was measured, to understand the processes governing the rate of microstructural change. Solid thin films at temperature too low allow morphology change by diffusion are necessarily metastable with respect to surface energy, since they have a high specific area. The observation that freshly deposited films were nonporous removed the possibility that a description of the energetics would have to take into account the energy of internal surface area. However, the role of surface energy had still to be considered among other possible driving forces for hillock formation. The growth of hillocks during the induction period cannot be regarded as driven by surface energy alone. The deposit is also affected by mechanical interaction with the substrate; during heating, different thermal expansion will cause the stress of the high expansivity metal to become more
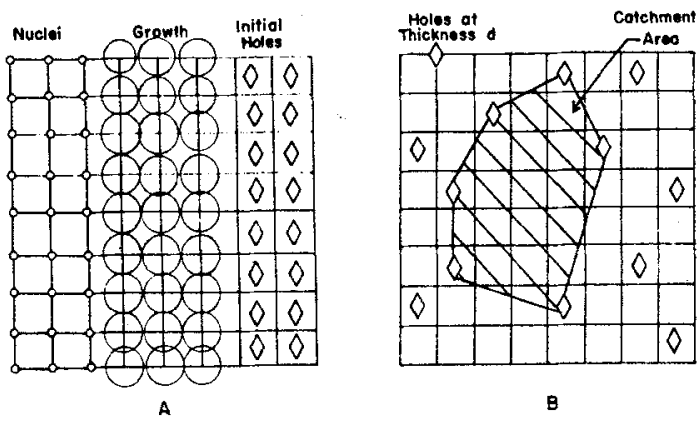

Figure 5. Delineation of the catchment area. 
compressive and this may lead to the formation of surface hillocks (Lahiri 1970; Pennebaker 1968; Chaudhuri 1969).

The transfer of material to the hillock above the surface proceeds until at the end of the induction period, the hole growth begins by withdrawal of metal in the remaining thin land areas from the substrate.

\section{Conclusions}

Several stages of discrete island formation during the annealing of thin gold films in oxygen containing atmospheres can be accounted for in terms of capillary theories of surface topography change of the type first proposed by Mullins (1957) for grain boundary greeving. The role of compression stress, suggested by other workers to contribute to initial hillock formation, is confirmed and the kinetics of hillock growth is accounted for in terms of surface diffusion transport. Subsequently, withdrawal of the material from the substrate is again by surface diffusion and the kinetics is governed by surface energy forces. The islands remaining at the end of the agglomeration process are shown to have drawn their material from areas delineated by the network of residual holes remaining after the film was initially deposited by heterogeneous nucleation and growth from the vapour.

\section{References}

Borel J P 1955 Mem. Soc. Vaudoise Sci. Nat. 112

Brandon R H and Bradshow F J 1966 R.A.E. (Farnborough) Rep. 66095

Caswell H L and Budo Y 1963 J. Appl. Phys. 343461

Caswell H L, Priest J R and Budo Y 1963 J. Appl. Phys. 343261

Chaudhuri P 1969 IBMi J. Res. Rep. 197

Gangulee A 1970 Philos. Mag. 22865

Gimpi M L, Mcmaster A D and Fuschille N 1964 J. Appl. Phj's. 353572

Lahiri S K 1970 J. Appl. Phys. 413172

Jorgenson G V and Wehner G K 1963 Trans. Tenth National Vacuum Symposium

Mullins W W 1957 J. Appl. Phys. 28355

Neilson N A, Keating K B and Miller W R 1967 Surface Sci. 8307

Pashley D W, Stewell M J, Jacibs M E and Law T W 1964 Philos. Mag. 10127

Pennebaker W B 1968 K.B.M. Res. Rep. RC-2105 